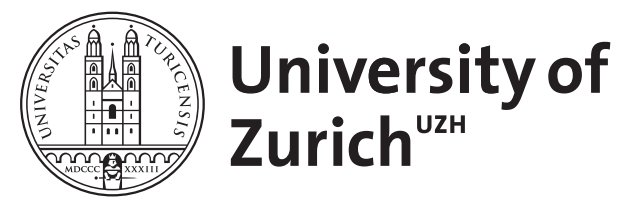

\title{
Computer-based lung sound simulation
}

\author{
Kompis, M ; Russi, E W
}

\begin{abstract}
An algorithm for the simulation of normal and pathological lung sounds is developed. The simulation algorithm is implemented on a personal computer as well as on a digital signal processor system in real time. Normal, bronchial and tracheal breathing sounds can be generated, and continuous and discontinuous adventitious lung sounds can be added. The attributes of the individual sound components, such as loudness, frequency, duration or number of occurrences within one breathing cycle, are controlled by the user. The quality of the simulations is evaluated by sending audio tapes to 15 experienced pulmonary physicians for a formal assessment. Each tape contains five simulated lung sounds and five real lung sounds from a commercially available teaching tape, presented in random order. Simulated lung sounds are slightly better rated in terms of realism and signal quality when compared to the recordings from the teaching tape. The differences are, however, not significant. 13 out of the 15 physicians feel that computer-based lung sound simulators would be a useful and desirable teaching tool for auscultation courses
\end{abstract}

DOI: https://doi.org/10.1007/bf02530043

Posted at the Zurich Open Repository and Archive, University of Zurich ZORA URL: https://doi.org/10.5167/uzh-156338

Journal Article

Published Version

Originally published at:

Kompis, M; Russi, E W (1997). Computer-based lung sound simulation. Medical Biological Engineering Computing, 35(3):231-238.

DOI: https://doi.org/10.1007/bf02530043 


\title{
Computer-based lung sound simulation
}

\author{
M. Kompis E. W. Russi \\ Pulmonary Division, Department of Internal Madicine, University Hospital of Zurich, 8091 Zurich,
} Switzerland

\begin{abstract}
An algorithm for the simulation of normal and pathological lung sounds is developed. The simulation algorithm is implemented on a personal computer as well as on a digital signal processor system in real time. Normal, bronchial and tracheal breathing sounds can be generated, and continuous and discontinuous adventitious lung sounds can be added. The attributes of the individual sound components, such as loudness, frequency, duration or number of occurrences within one breathing cycle, are controlled by the user. The quality of the simulations is evaluated by sending audio tapes to 15 experienced pulmonary physicians for a formal assessment. Each tape contains five simulated lung sounds and five real lung sounds from a commercially available teaching tape, presented in random order. Simulated lung sounds are slightly better rated in terms of realism and signal quality when compared to the recordings from the teaching tape. The differences are, however, not significant. 13 out of the 15 physicians feel that computerbased lung sound simulators would be a useful and desirable teaching tool for auscultation courses.
\end{abstract}

Keywords-Auscultation, Computer simulation, Computer-aided instruction, Respiratory sounds, Teaching materials

Med. Biol. Eng. Comput., 1997, 35, 231-238

\section{Introduction}

CHEST AUSCULTATION is an important part of clinical patient assessment. The skills required to interpret the auscultatory findings are traditionally learned by listening to the lung and heart sounds of many different patients. Although this method is excellent in terms of a comprehensive approach to the patient, it is afficted with some inherent disadvantages. It may be difficult for a student to compare actual lung or heart sounds with similar findings, since they may not be accessible at the same time. Also, rare auscultatory findings are seldom available for demonstration.

For lung sounds, tape recordings of real auscultatory findings solve only a part of the above-mentioned problems. The instructor has to rely on a limited collection of lung sounds, whereby the duration of each sound is itself limited. For comparing different auscultatory findings, tape recordings are not a satisfactory solution, since the recordings often differ in several parameters in concert such as loudness, location in the breathing cycle and timbre, and hardly ever only in that one aspect which is in the focus of interest.

Computer-aided instructional programs (RUSSO, 1991) based on digital signal presentation (PASTERKAMP et al., 1989) are superior to tape recordings in a number of aspects, such as speed of access to any desired recording or the availability of airflow information. However, some of the drawbacks persist, most notably the inability to vary a single aspect of a lung sound while keeping all others unchanged.

First received 19 April and in final form 13 November 1996 Correspondence should be addressed to Erich W. Russi

TIFME: 1997
Heart sound simulators (TRAN et al., 1995) have been used for many years and are able to overcome many of these problems. The use of these devices for teaching purposes has been investigated systematically and shown to be beneficial (HARREL et al., 1990). To our knowledge, no similar device exists for the simulation of lung sounds. Apart from their use as a teaching tool, lung sound simulators might be useful in all applications which require well-defined and easily reproducible lung sounds, such as the evaluation of computerised lung sound analysis systems (COHEN and LANDSBERG, 1984; KAISLA et al., 1991; NISSAN and GAVRIELY, 1993; SANKUR et al., 1994).

We aimed to assess the feasibility and potential usefulness of a computer-based lung sound simulator. This was accomplished by developing a new flexible simulation algorithm followed by a blinded study to compare the quality of the simulated sounds to those recorded on a commercially available teaching tape (BAEDEKER et al., 1991).

\section{Lung sound simulation algorithm}

Fig. 1 shows a block diagram of the lung sound simulation algorithm. Simulated signals are generated as sequences of samples at a constant sampling rate of $2500 \mathrm{~Hz}$. This rate is low when compared to the rates usually used in lung sound analysis (COHEN and LANDSBERG, 1984; KAISLA et al., 1991; SANKUR et al., 1994; PASTERKAMP, 1992), but is sufficient to represent most of the signal energy of the majority of all lung sounds. In contrast to lung sound analysis, no unexpected high-frequency components can occur, and computing time can be economised. In principle, the presented algorithm can generate simulated lung sounds at any sampling rate, where 


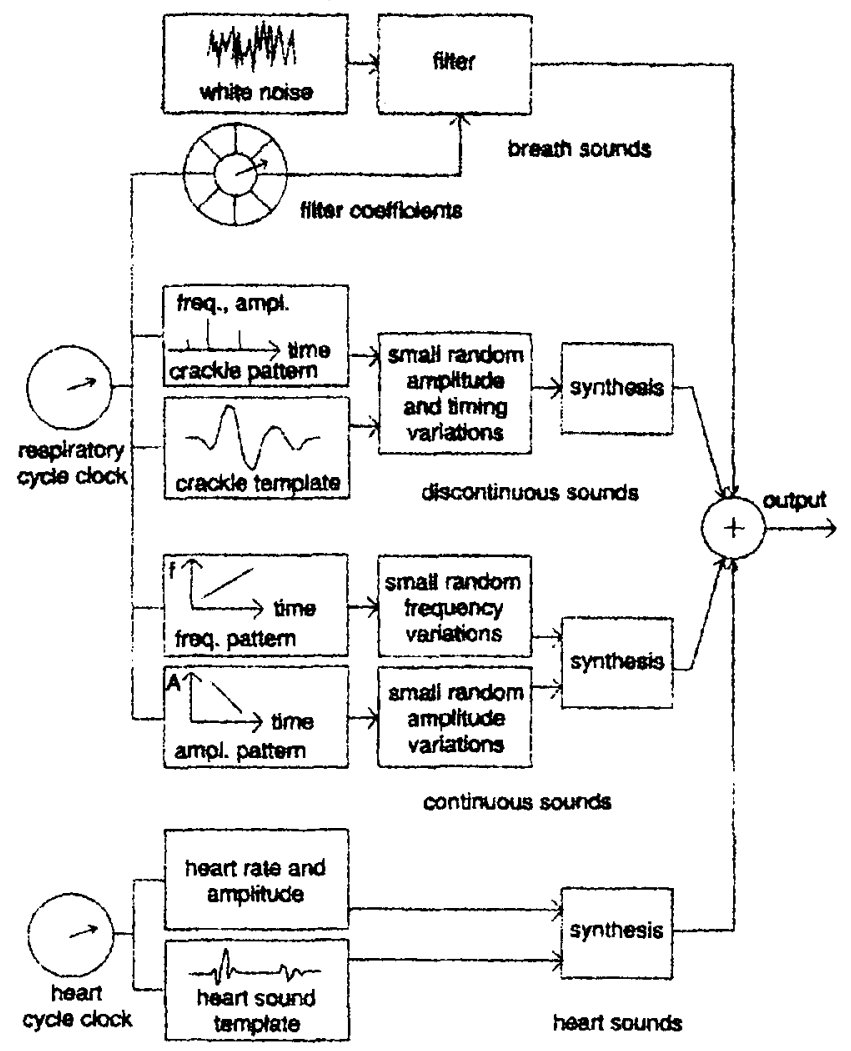

Fig. 1 Schematic representation of lung sound simulation algorithm

low values will mainly limit the reproduction of the highfrequency components of tracheal sounds.

When analysing lung sound recordings, several sound categories can be discriminated. The presented simulation algorithm can generate signals containing sounds from four different sound categories, as shown in Fig. 1. Three categories of lung sounds, namely breath sounds, continuous adventitious sounds and discontinuous adventitious sounds, as well as heart sounds, can be generated. Breath sounds, discontinuous and continuous adventitious lung sounds are linked by a common respiratory cycle clock. Other than that, each category is generated independently, and any or all of them can be deactivated by the user.

\subsection{Breath sounds}

Breath sounds can be characterised as band-limited noise, the amplitude and spectrum of which changes in a characteristic manner as a function of the air-flow rate over a respiratory cycle. Depending on the auscultation site and the underlying pathology (if any), breath sounds can be normal, bronchial, or tracheal, and are classified according to their spectral characteristics (GAVRIELY, 1995; KoMPIS, 1995). Signal 1 in Fig. 2 shows an example of a normal breath sound, whereas signal 4 shows a tracheal breath sound along with some heart sounds. As a linear grey-scale is used in Fig. 2 , there seems to be a relatively sudden drop in the spectra above $450 \mathrm{~Hz}$ and $650 \mathrm{~Hz}$, respectively.

Breath sounds are imitated by filtering white noise produced by a random number generator (WIDROW and STEARNS, 1985) with an all-pole infinite impulse response filter. The filter coefficients are averaged estimates of auto-regressive (AR) parameters of real lung sounds. The characteristics of the simulated sound is similar to the real sound used for analysis, so filter-sets for normal, bronchial and tracheal breath sounds were generated. It was observed previously that at the given sampling rate the information of no more than four $A R$ - coefficients can be found in successive breath cycles. (KompIs, 1995). Thus a filter order of four was used, which is in the lower range when compared to the extreme values of 3-4 (VANDERSCHOOT et al., 1992) and 6-8 (GAVRIELY and HERZBERG, 1992) found in the literature with different sampling rates and quality criteria.

Although frequency spectra have been found to change little for certain airflow ranges and recording sites (KRAMAN, 1986; GAVRIELY and CUGELL, 1996), our own findings and published data (GAvriely and CuGELL, 1996, Figs. 2B and 5) suggest that amplitudes increase faster with airflow at frequencies above $300 \mathrm{~Hz}$ than at lower frequencies. This makes the use of multiple sets of coefficients advisable. 16 sets of filter coefficients, stored in a cyclic table, are used to model a single respiratory cycle; 8 each for inspiration and expiration. This number of sets was selected as it was found that further segmentation of real breath sounds does not increase the stationarity within the segments (Reverse arrangement test: GAVRIELY and HERZBERG (1992); BENDAT and PIERSOL, (1986)). The actual filter coefficients are taken from the cyclic table according to the momentary state of the respiratory cycle clock. Transitions between successive filter sets are smoothed by linear interpolation of the filter coefficients. Inspiration is assumed to take up $30 \%$ of the total respiratory cycle time, expiration $70 \%$. However, this ratio can be changed by the user.

\subsection{Discontinuous adventitious lung sounds}

Discontinuous adventitious lung sounds or crackles are short acoustic events of less than $20 \mathrm{~ms}$ duration, consisting of an initial deflection followed by a number of oscillations. Crackles can be heard in a variety of pathologic conditions. They can be classified as coarse or fine according to their temporal and spectral characteristics (MUNAKATA et al., 1991). Often the distribution of the crackles within the respiratory cycle follows a pattern which can be found in several successive breaths.

To model discontinuous adventitious sounds, a crackle pattern table is generated under the control of a random number generator. The table contains the timing and the coarseness of the number of crackles chosen by the user. The template of a single coarse crackle from a real lung sound recording (five half cycles, total duration $7.2 \mathrm{~ms}$, initial deflection width IDW $1.5 \mathrm{~ms}$, two cycle duration $2 \mathrm{CD}$ $6.2 \mathrm{~ms}$, see MUNAKATA et al., (1991) for definitions) is stored in a table. Crackles with different degrees of coarseness are produced by compression of the crackle template in the time domain. Table 2 in the text of Munakata et al., (1991) supports the underlying assumption that fine and coarse crackles can be modelled with a reasonable accuracy using a single, accordingly compressed or stretched waveform. The resulting waveform is added to the simulated signal. As $99 \%$ of the signal energy of the crackle template can be found below $650 \mathrm{~Hz}$, compression factors of up to 1.9 can be used at the given sampling rate of $2500 \mathrm{~Hz}$. For enhanced realism, small random variations in amplitude $( \pm 20 \%)$ and timing ( $\pm 20 \mathrm{~ms}$ ) of the individual crackles make the patterns in successive breath cycles similar, but not identical.

\subsection{Continuous adventitious lung sounds}

Continuous adventitious lung sounds can be heard in various pathologic conditions, most notably in asthma. They are almost periodic with a fundamental frequency of usually between 100 and $800 \mathrm{~Hz}$ and durations mostly between 0.3 and $2 \mathrm{~s}$ (KOMPIS, 1995). Signals 9 and 10 in Fig. 2 show two 

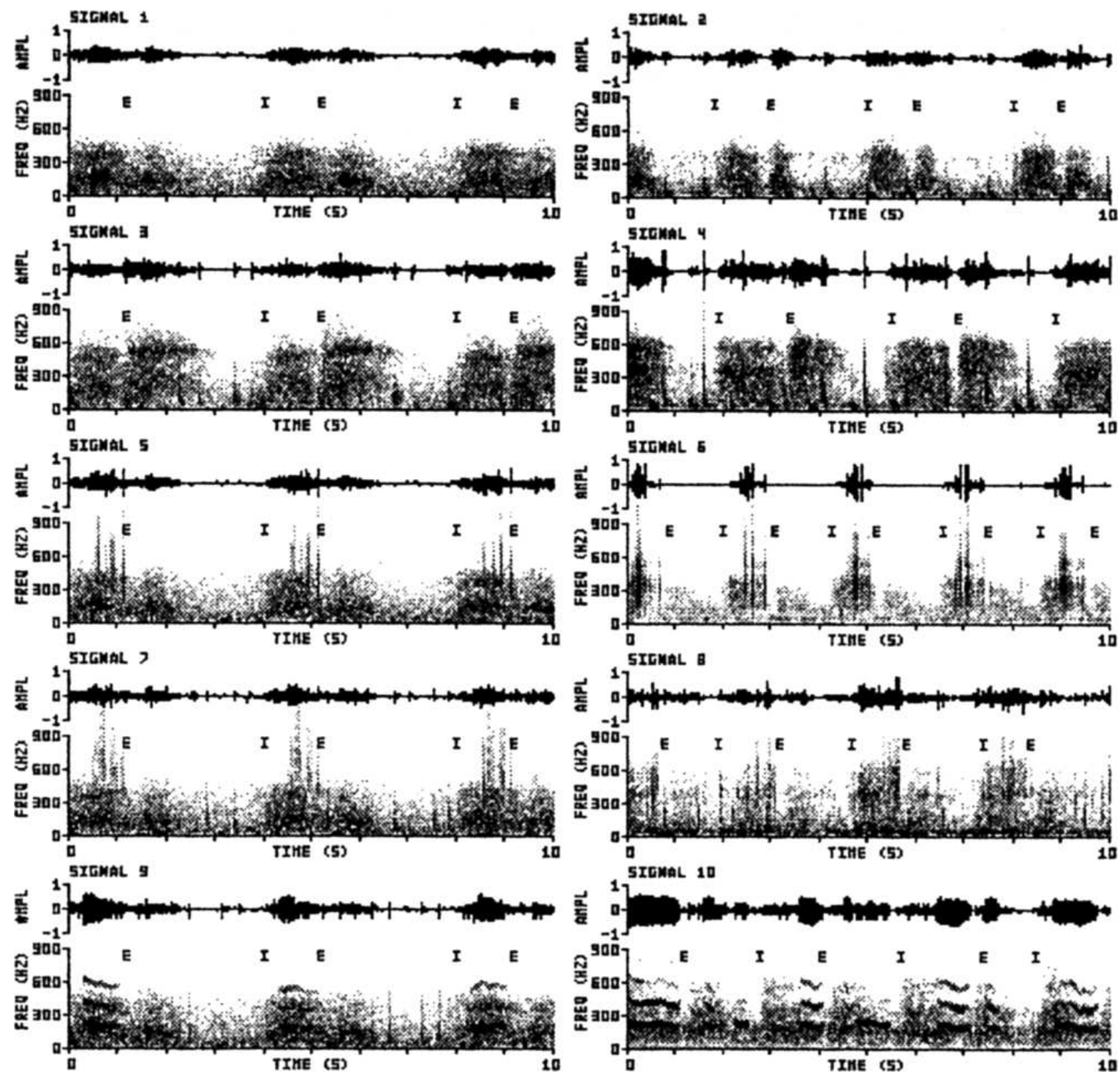

Fig. 2 Representative sections of $10 \mathrm{~s}$ of the ten signals used in the evaluation study. The upper trace in each signal representation shows the amplitude (AMPL) in arbitrary units vs. time. The lower portion shows the corresponding sonogram, i.e. the magnitude (arbitrary units, same linear greyscale for all signals) plotted against time and frequency. I and $E$ denote the beginning of inspiration and expiration, respectively

examples of lung sounds containing continuous adventitious sounds.

To simulate continuous adventitious lung sounds, manually generated tables containing the coarse amplitude and frequency pattern are used. These basic patterns contain only the relative pitches and amplitude levels at the beginning and at the end of a given continuous adventitious lung șound and are modified by the user in absolute pitch, absolute loudness, duration, as well as by small random amplitude and frequency variations under the control of a random number generator. It was found that small variations of both amplitude and frequency are necessary to obtain a reasonably natural sound. Continuous adventitious sounds $s(t)$ ( $t$ denotes time) are synthesised by evaluating the formula

$$
\begin{aligned}
s(t)= & A(t) \cdot\left(\sin (\omega(t) \cdot t)+A_{1} \cdot \sin (2 \omega(t) \cdot t)\right. \\
& \left.+A_{2} \cdot \sin (3 \omega(t) \cdot t)\right)
\end{aligned}
$$

with fixed values $A_{1}=0.5$ and $A_{2}=0.1$. The amplitude $A(t)$ and the momentary radian frequency $\omega(t)$ are calculated as

$$
A(t)=A_{0}(t) \cdot C_{A}(t)
$$

and

$$
\omega(t)=2 \pi \cdot f_{0}(t) \cdot C_{F}(t)
$$

respectively. $A_{0}(t)$ denotes the coarse amplitude pattern and $f_{0}(t)$ the frequency pattern from the above-mentioned tables. $C_{A}(t)$ and $C_{F}(t)$ are slowly fluctuating functions with an expected value of 1 , each evaluated separately using the formula

$$
C\left(t+T_{s}\right)=1+(C(t)-1) \cdot \sqrt{1-\frac{T_{s}}{T_{R}}}+A_{R} \cdot R \cdot \sqrt{\frac{T_{s}}{T_{R}}}
$$


where $T_{y}$ denotes the sampling period and $R$ is a different number from a zero-mean random-number sequence each time eqn. 4 is evaluated. $C(t)$ shows slow random variations around 1 with a time constant of $T_{R}$ and a standard deviation of $A_{R}$ times the standard deviation of the random number sequence from which $R$ is taken. In our simulation, $R$ has a uniform probability density distribution between -1 and $+1 . A_{R}$ is 0.1 for the frequency variations and 0.4 for amplitude variations. A time constant $T_{R}$ of $0.25 \mathrm{~s}$ is used for all calculations.

\subsection{Heart sounds}

To simulate heart sounds, two tables holding the template of a first heart sound and a second heart sound are stored. Versions of the templates, scaled in amplitude, are added to the simulated signal according to the heart rate and loudness desired by the user. This simple procedure does not tum the lung sound simulator into a heart sound simulator, but it can add an astonishingly realistic touch to the simulated output signal.

\section{3 implementation}

The lung sound simulation algorithm was implemented on two different platforms. In the version used for the evaluation study in Section 4, the simulator is a FORTRAN program for personal computers. Signals of up to $30 \mathrm{~s}$ in length can be created off-line, i.e. the signal is available only after the entire process of simulation has been completed. In a second version, a slightly simplified form of the algorithm was implemented on a ADSP2101 digital signal processor system*, in real time using assembly language. Changes of the simulation parameters during the simulation process are immediately effective and determine the properties of the continuously generated lung sound.

\section{Evaluation study of quality of simulations: materials and methods}

The primary goal of lung sound simulation is to produce a clear and realistic subjective impression in the learning process. Therefore, the evaluation of the simulation must be based on the subjective judgement of human listeners. Although objective criteria such as the frequency spectra must be similar for similar real and simulated signals, they do not by themselves guarantee an adequate subjective impression in a human listener.

To evaluate the quality of the simulated sounds, audio tapes with real and simulated lung sounds were sent to 15 pulmonary physicians for a formal assessment. Their clinical experience at the time of the investigation ranged from 14 to 30 years (mean 18.6 years).

\subsection{Test signals}

For the recording of the audio tapes, a personal computer equipped with a 12 bit digital-to-analogue converter was used. The sampling rate was $2500 \mathrm{~Hz}$. The signals were passed through a low-pass filter with a cutoff frequency of $1160 \mathrm{~Hz}$ and $72 \mathrm{~dB} /$ octave roll off, prior to recording on audio tapest.

Each tape contained the same ten lung sounds, but their order was varied randomly. Table 1 shows a list of these

* Espresso-board, Saddle Point Systems, Oakland CA, USA

+ Sony C60 UX-S tapes, Technics RS-X101 tape recorder sounds. Five real lung sounds from a commercially avaitable teaching tape (BAEDEKER et al., 1991) were recorded along with five simulated sounds. The duration of each sound was 30 s. The signal-to-noise ratio (SNR) was estimated from the signals which do not contain adventitious lung sounds (signals 1 to 4) by evaluating the total signal variance in consecutive segments of $0.1 \mathrm{~s}$. The variance of those $10 \%$ of all segments with the lowest variance (end-expiratory segments considered to contain background noise only) was then compared with the variance of the loudest $10 \%$. The SNRs were $16.5 \mathrm{~dB}$ and $17.1 \mathrm{~dB}$ (simulated and real sound, respectively) for normal breath sounds and $19.8 \mathrm{~dB}$ and $19.4 \mathrm{~dB}$, respectively, for tracheal sounds. Fig. 2 shows a representative portion of $10 \mathrm{~s}$ of each sound in the time domain and in a sonogram representation. For the simulations, we attempted to maintain the highest possible grade of realism, while matching the acoustic impression of the teaching tape examples. For all simulated sounds, an entire respiratory cycle was $4 \mathrm{~s}$ long, the inspiratory part being 30\% thereof. Signal 5 (coarse crackles), was simulated with five crackles per inspiration (compression of the crackle template in the time domain by 0.9 ), signal 7 (fine crackles) with 50 crackles (time compression factor 0.6 ). In signal 9, each continuous adventitious sound lasted for $0.85 \mathrm{~s}$. The underlying coarse frequency pattern was a constant fundamental frequency $f_{0}$ of $180 \mathrm{~Hz}$, and the relative amplitude pattern function $A_{0}(t)$ was a linearly decreasing slope starting at 1.0 and ending at 0.5 within each continuous sound.

\subsection{Questionnaire}

The physicians were informed that real and simulated signals were recorded on the tapes, but not how many of which category and in which order. They had to judge each signal using a multiple choice questionnaire on the following aspects:

(i) the clinical finding

(ii) the realism of the signal and the assumed origin (simulation or real signal)

(iii) the overall quality of the signal

As a minimal requirement, the output signal of a useful lungsound-simulator must be clearly and unequivocally recognised by a trained physician. To describe the clinical finding the physicians were asked to rate the breath sound either as normal, tracheal or of unclear quality. Discontinuous and

Table I Signals recorded on the audio tapes used in the evaluation sindy

\begin{tabular}{|c|c|c|c|}
\hline \multicolumn{2}{|c|}{ simulated sounds } & \multicolumn{2}{|c|}{$\begin{array}{l}\text { real sounds from the teaching } \\
\text { tape }\end{array}$} \\
\hline signal no. & description & signal no. & description \\
\hline 1 & $\begin{array}{l}\text { normal breath } \\
\text { sound }\end{array}$ & 2 & $\begin{array}{l}\text { normal breath } \\
\text { sound }\end{array}$ \\
\hline 3 & $\begin{array}{l}\text { tracheal breath } \\
\text { sound with loud } \\
\text { heart sounds }\end{array}$ & 4 & $\begin{array}{l}\text { tracheal breath } \\
\text { sound with loud } \\
\text { heart sounds }\end{array}$ \\
\hline 5 & $\begin{array}{l}\text { coarse late } \\
\text { inspiratory crackles }\end{array}$ & 6 & $\begin{array}{l}\text { coarse late } \\
\text { inspiratory crackles }\end{array}$ \\
\hline 7 & $\begin{array}{l}\text { fine late inspiratory } \\
\text { crackles with soft } \\
\text { heart sounds }\end{array}$ & 8 & $\begin{array}{l}\text { fine late inspiratory } \\
\text { crackles with soft } \\
\text { heart sounds }\end{array}$ \\
\hline 9 & $\begin{array}{l}\text { inspiratory rhonchi } \\
\text { with soft heart } \\
\text { sounds }\end{array}$ & 10 & $\begin{array}{l}\text { ins- and expiratory } \\
\text { thonchi and } \\
\text { wheezes with soft } \\
\text { heart sounds }\end{array}$ \\
\hline
\end{tabular}


continuous adventitious sounds had to be categorised either as present or absent. In addition, if discontinuous sounds were perceived, it was asked whether they were fine or coarse.

To determine the realism of the presented lung sounds the participants were asked to judge the realism and the assumed origin of each signal by choosing one of the following:

-natural sound/probably real lung sound

-intermediate realism/undecided about the source

-artificial sound/probably simulated lung sound

The answers were formulated in such a way that the realism of each sound was linked explicitly to the assumed source. This measure was taken to avoid puzzling combinations.

The physicians rated the overall signal quality either as good, intermediate or poor. To answer this question, they were asked to rely on their subjective judgment only. No additional criteria were given, as how to judge the rather vague term of signal quality. This approach was chosen to obtain a sensitive parameter, where the participants could show their uneasiness if they felt that there was 'something wrong' with a signal, possibly without being able to be more specific. In an additional question, the participants were asked whether they thought that using a lung sound simulator for teaching purposes would be useful and desirable.

\subsection{Statistical analysis}

The statistical significance of the differences between real and simulated lung sounds was evaluated on a $5 \%$ level by one-sided Wilcoxon-Mann-Whitney test.

\section{Evaluation study of quality of simulations: results}

\subsection{Clarity of the findings}

To assess the clarity of the findings, the distribution of serious mistakes in the judgments of the findings was analysed. A serious mistake was assumed, when either:

(i) an adventitious continuous or discontinuous lung sound was not heard, although it was present in the signal or

(ii) an adventitious continuous or discontinuous lung sound was perceived, although there was none in the signal or

(iii) an adventitious continuous sound was perceived as a discontinuous sound or vice versa.

In the judgement of the 75 presented simulated signals, nine serious mistakes occurred, whereas in the 75 real signals from the teaching tapes, 11 serious mistakes occurred. Table 2 shows the distribution of these mistakes.

\subsection{Realism and signal quality}

On the left of Fig. 3, the rating of sound realism is shown for 147 of the 150 presented signals. Three answers (one each of the signals 2, 3 and 4 in Table 1) were not usable, i,e. no or several answers were given. The simulated signals were rated as slightly more natural than the signals from the teaching tape, however, the difference was not statistically significant.

The right side of Fig. 3 shows a similar representation for rating of the sound quality for 148 of the 150 presented signals. Two answers were not usable. The signal quality of $66.7 \%$ of the real and $70.7 \%$ of the simulated signals was rated as good. The simulated signals were perceived as slightly better in quality, although again the difference was not statistically significant.
Table 2 Distribution of serious mistakes

\begin{tabular}{|c|c|c|}
\hline description of mistake & $\begin{array}{c}\text { number of } \\
\text { occurrences in } \\
\text { simulated signals }\end{array}$ & $\begin{array}{l}\text { number of } \\
\text { occurrences in real } \\
\text { lung sound }\end{array}$ \\
\hline $\begin{array}{l}\text { discontinuous adventitious } \\
\text { lung sounds were } \\
\text { perceived, although none } \\
\text { were present in the signal }\end{array}$ & $\begin{array}{l}4 \text { out of } 45 \\
(8.9 \%)\end{array}$ & $\begin{array}{l}9 \text { out of } 45 \\
(20 \%)\end{array}$ \\
\hline $\begin{array}{l}\text { continuous adventitious } \\
\text { lung sounds were } \\
\text { perceived, although none } \\
\text { were present in the signal }\end{array}$ & $\begin{array}{l}4 \text { out of } 60 \\
(6.7 \%)\end{array}$ & $\begin{array}{l}2 \text { out of } 60 \\
(3.3 \%)\end{array}$ \\
\hline $\begin{array}{l}\text { continuous or } \\
\text { discontinuous adventitious } \\
\text { lung sounds were not } \\
\text { perceived, although they } \\
\text { were present in the signal }\end{array}$ & $\begin{array}{l}0 \text { out of } 45 \\
(0.0 \%)\end{array}$ & $\begin{array}{l}0 \text { out of } 45 \\
(0.0 \%)\end{array}$ \\
\hline $\begin{array}{l}\text { discontinuous adventitious } \\
\text { lung sounds were perceived } \\
\text { as continuous lung sounds }\end{array}$ & $\begin{array}{l}\text { I out of } 30 \\
(3.3 \%)\end{array}$ & $\begin{array}{l}0 \text { out of } 30 \\
(0.0 \%)\end{array}$ \\
\hline
\end{tabular}

\subsection{Realism and discernibility of individual sound compo- nents}

The perceived realism was also analysed as a function of the individual sound components contained in the presented signals. Fig. 4 shows the results for the breath sounds only (signals 1,2,3, and 4), for the signals with discontinuous adventitious lung sounds (signals $5,6,7$, and 8 ), and for the signals with continuous adventitious lung sounds (signals 9 and 10 ). For all three categories, the simulated sounds were rated as slightly more natural. Again, none of the differences was statistically significant.

Besides realism, the discernibility between normal and tracheal breath sounds as well as between fine and coarse crackles was analysed. For the discernibility between normal and tracheal breath sounds, only the answers concerning signals $1,2,3$, and 4 were used. Although all signals contained breath sounds, its quality was not explicitly stated for those teaching-tape examples, which contained adventitious lung sounds. Furthermore, it was assumed that the participants might have been distracted by adventitious lung sounds. The results in Table $3 a$ show that $80 \%$ of the simulated breath sounds and $67 \%$ of the real breath sounds were rated correctly.

The analysis of discemibility between fine and coarse crackles was performed with signals $5,6,7$, and 8 . The results are summarised in Table $3 b$. In $80 \%$ of the simulated signals and $60 \%$ of the real signal the crackles were classified correctly.

\subsection{Usefulness of lung sound simulator for teaching purposes}

The participants were asked whether they thought that using a lung sound simulator for teaching purposes would be useful and desirable. The four possible answers were (i) yes, (ii) no, (iii) I don't know; I am not sure, and (iv) I do not feel competent enough to answer this question. 13 out of the 15 physicians $(86.7 \%)$ thought that lung sound simulators would be useful (yes) and two of them (13.3\%) were not sure (answer iii).

\section{Discussion}

A flexible lung sound simulation algorithm was developed and implemented. The quality of the simulations was evaluated in a blinded study. Our findings suggest that simulated 

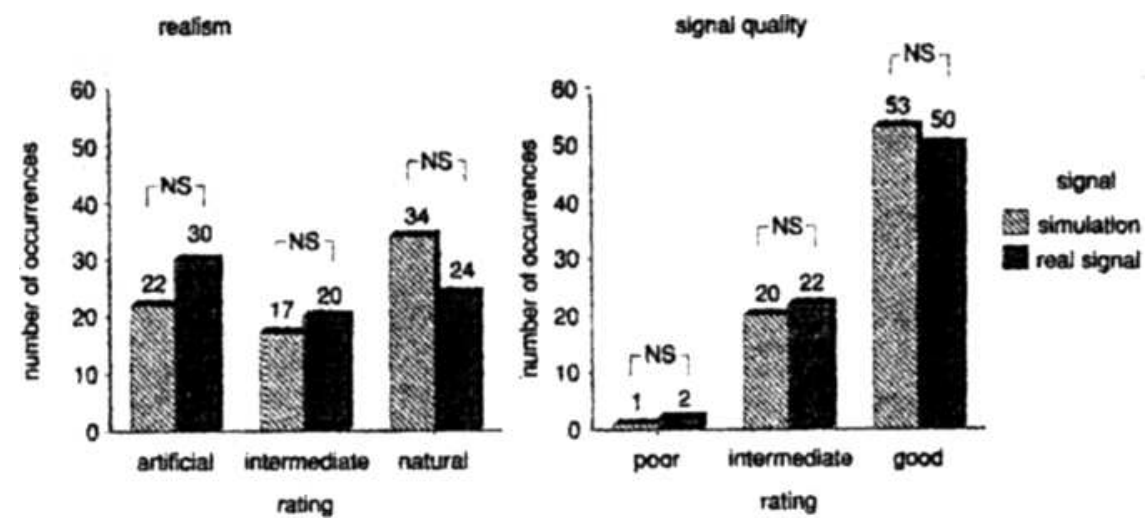

Fig. 3 Rating of presented signals in terms of realism (left) and signal quality (right)
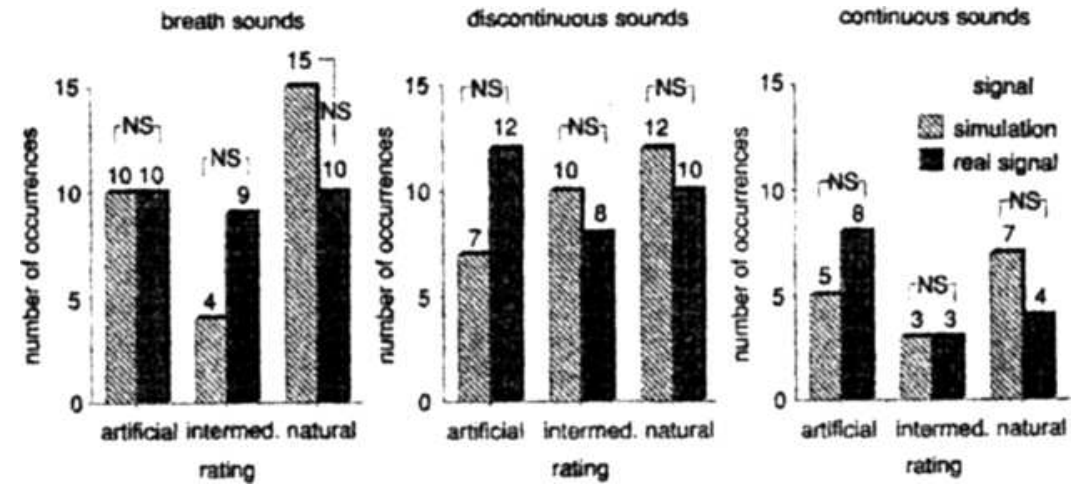

Fig. 4 Rating of the realism of individual signal components. Breath sound data summarises the ratings of signtals 1 to 4 in Table 1; discontinuous sounds signals 5 to 8, and continuous sounds signals 9 and 10

lung sounds can be as discernible and natural as their real counterparts. In all aspects which were evaluated by experienced chest physicians, the simulated lung sounds were perceived as slightly superior to the real ones, though none of these differences were statistically significant

In the evaluation study, a commercially available teaching tape was used. This choice was motivated as follows. The tape is widely used by students and thus takes a similar position in learning auscultation as the simulator is planned to do. The recordings are very typical for a variety of specific ausculta-

Table $3 a$ Discemibility between normal and tracheal breath sounds

\begin{tabular}{lccccc}
\hline rating & \multicolumn{2}{c}{$\begin{array}{c}\text { answers for simulated } \\
\text { signals }\end{array}$} & & \multicolumn{2}{c}{$\begin{array}{c}\text { answers for real lung } \\
\text { sounds }\end{array}$} \\
\cline { 2 - 3 } \cline { 6 - 7 } & $\begin{array}{c}\text { normal } \\
\text { breath }\end{array}$ & $\begin{array}{c}\text { tracheal } \\
\text { breath }\end{array}$ & & $\begin{array}{c}\text { normal } \\
\text { breath }\end{array}$ & $\begin{array}{c}\text { tracheal } \\
\text { breath }\end{array}$ \\
\hline normal & 12 & 1 & & 8 & 1 \\
tracheal & 0 & 12 & & 3 & 12 \\
unclear & 3 & 2 & & 4 & 2 \\
\hline
\end{tabular}

Table $3 b$ Discernibility between fine and coarse crackles

\begin{tabular}{lccccc}
\hline rating & \multicolumn{2}{c}{$\begin{array}{c}\text { answers for simulated } \\
\text { signals }\end{array}$} & & \multicolumn{2}{c}{$\begin{array}{c}\text { answers for real lung } \\
\text { sounds }\end{array}$} \\
\cline { 2 - 3 } \cline { 6 - 7 } & $\begin{array}{c}\text { fine } \\
\text { crackles }\end{array}$ & $\begin{array}{c}\text { coarse } \\
\text { crackles }\end{array}$ & & $\begin{array}{c}\text { fine } \\
\text { crackles }\end{array}$ & $\begin{array}{c}\text { coarse } \\
\text { crackles }\end{array}$ \\
\hline fine & 12 & 2 & & 9 & 6 \\
coarse & 2 & 12 & & 6 & 9 \\
no answer & 1 & 1 & & 0 & 0 \\
\hline
\end{tabular}

tory findings, and their quality (i.e. background noise, artifacts, timbre when compared to auscultation by stethoscope) is excellent when assessed in informal listening tests. One drawback of the teaching tape is that detailed information on the recording apparatus is not readily available. However, we feel that for a subjective comparative study the excellent reproducibility of the sounds themselves more than outweighs the limited reproducibility of the original recording conditions.

When analysing the clarity of the findings (Table 2), it is noteworthy that out of a total of 20 serious mistakes, 19 involved an adventitious lung sound which was heard where none was present. Most of the errors concentrated on only a few signals. Six out of the 15 physicians believed they heard crackles in the normal breath sound from the teaching tape (signal 2), and three of them heard a continuous adventitious sound in the simulated tracheal breath sound (signal 3). 15 of the non-existing adventitious sounds were heard in signals without a pathological finding (signals 1 to 4). We believe that these serious mistakes can partly be explained as a systematic bias caused by the study design. Some of the participants might have suspected that a pathological finding is present in most of the presented signals. We analysed signal 1 by visual inspection (time domain and sonogram) as well as by listening to it through high-as well as low-quality tape recorders. No evidence was found for the presence of crackles. Soft heart sounds are present, but to our judgement, they cannot be confused with crackles. Nevertheless, it is impossible to reject the possibility that any given real hung sound contains absolutely no very sof adventitious lung sounds.

The continuous sounds heard in signal 3 can be explained by the prominent frequency band around $500 \mathrm{~Hz}$ (see Fig. 2). Possibly, this undesired feature in our simulations could be diminished by choosing a different tracheal breath sound for the calculation of the filter coefficients or by using a higher 
filter order for tracheal sounds, as suggested by GAVRIELY and HERTZBERG, (1992). Tracheal sounds are known to contain signal energy even above $1500 \mathrm{~Hz}$. Although we think that the spectral maximum around $600-700 \mathrm{~Hz}$ may be the key feature by which human listeners discriminate between normal and tracheal breath sounds, there are other maxima, namely around $1500 \mathrm{~Hz}$ (KRAMAN et al., 1996) which are not reproduced with the sampling rate of $2500 \mathrm{~Hz}$. As the required computing power is by now very affordable, we suggest that future implementations of the lung sound simulator use sampling rates well above $3 \mathrm{kHz}$.

The initial deflection width IDW was $1.4 \mathrm{~ms}$ for the simulated coarse crackles, and $0.9 \mathrm{~ms}$ for the simulated fine crackles. The two cycle duration for these signals was $5.6 \mathrm{~ms}$ and $3.7 \mathrm{~ms}$, respectively. In the corresponding real signals, these values varied slightly, but were bound to be very similar on average, as the choice of the simulation parameters was based upon them. The coarse and fine crackles in our test material sound distinctly different, and the misclassification rate of 20 to $40 \%$ between coarse and fine crackles is probably due to the different personal interpretations of the terms 'coarse' and 'fine'. Note that the impression of a relative lack of low-frequency signal energy in signal 8 (real coarse crackles, Fig. 2) results from the combination of soft breath sounds and loud crackles with their inherent bandpass characteristics.

When comparing continuous adventitious lung sounds (diagram on the right hand side in Fig. 4), the difference between test signals 9 and 10 was greater than in the other examples, signals 1 to 8 . The real signal contained several inspiratory and expiratory continuous sounds, whereas in the simulated signal a single inspiratory wheezing sound per breathing cycle was present. This single sound was similar in pitch to those in the real signal. Although several adventitious lung sounds could have been easily simulated, we chose not to overload the signal as the result seemed more natural to us. It is possible and even likely that the participants felt the same way when they judged the two signals. If this is true, then it was the composition of the signal that was favoured in the simulation rather than the naturalness of the individual signal components. The capability of composing a lung sound according to individual needs is a unique and desirable feature of a simulator.

While some of the parameters used for simulation are based on previous research or our systematic investigations (KOMPIS, 1995), many of the parameters represent what we consider to be reasonable estimates. This is especially true for the simulation of continuous adventitious lung sounds, where much of the required data is not readily available today. We do not claim to have found or used the best algorithms or parameters for lung sound simulation. However, we believe that they are sufficient to prove the feasibility of computer-based lung sound simulators and to show their potential.

With the given simulation algorithm, it is possible to simulate lung sounds which are comparable to actual sounds with respect to realism. It is also possible to create quite unnatural sounds, e.g. by exaggerating one of the simulating parameters. This may be a risk of the simulator, and in a clinical teaching module, a waming mechanism might be useful to alert the user about unrealistic parameters settings. However, the possibility of exaggerating parameters is a major advantage of the simulator. When used as a teaching tool, any aspect can be emphasised, until it is clearly audible and discernible from other acoustic elements, even to the inexperienced listener.

13 out of the 15 physicians indicated that a lung sound simulator would be a useful instrument for teaching purposes. This result emphasises one potential application. Lung sounds simulators might be used in auscultatory classes or as one of the features in computer-aided instruction (CAI) programs (e.g. MANGione et al., 1992). They can be easily embedded into multimedia presentation settings (SESTINI et al., 1995). Apart from their use as a teaching aid, lung sound simulators might be useful for other applications where well-defined and easily reproducible lung sounds are required. These applications might include investigations into the capabilities and limits of individual listeners for lung sound discrimination, or testing and evaluation of automatic lung sound analysis systems (COHEN and LANDSBERG, 1984; KaISLA et al., 1991; NISSAN and GAVRIELY, 1993; SANKUR et al., 1994). As the simulator was designed and tested only as a source of signals for human listeners, it is possible that the simulations would not show the same degree of naturalness when analysed by complex computer programs.

Acknowledgements-This work was supported by the Roche Research Foundation, Basle (Switzerland), the Janggen-Pöhn Foundation, St. Gallen (Switzerland), and the Emst-Hadom Foundation, Zurich (Switzerland). The authors wish to thank the publishing house Urban und Schwarzenberg. Munich (Germany), for their permission to use their teaching tape (BAEDEKER et al., 1991) for our study, and Prof. G. R. Wodicka and Dr. K. Bloch for their critical advice and help in preparing this text.

\section{References}

Baedeker, W., Kroker, P. B., Sybrecht, G. W., and Strufe, R. (1991): 'Herztöne und Lungengeräusche' (Urban Schwarzenberg, Munich, Germany)

Bendat, J., and Piersol, A. (1986): 'Random Data' (Wiley, 2nd Edn.) pp. 97-99

COHEN, A., and LANDSBERG, D. (1984): 'Analysis and automatic classification of breath sounds', IEEE Trans. Bio-Med. Eng., 33, pp. $585-590$

GAVRIELY, N., and HERZBERG, M. (1992): 'Parametric representation of normal breath sounds', $J$. Appl. Physiol., 73, pp. 1776-1784

Gavriely, N. (1995): 'Breath sounds methodology' (CRC Press, Florida, USA)

Gavriely, N., and Cugell, D. W. (1996): 'Airflow effects on amplitude and spectral content of normal breath sounds', $J$. Appl. Physiol., 80, pp. $5-13$

Harrel, J. S., Champagne, M. T., Jarr, S., and MiYaya, M. (1990): 'Heart sound simulation: how useful for critical care nurses?', Hean Lung, 19, pp. 197-202

Kaisla, T., Sovuärvi, A., Purlä, P., Rajala, H. M., haltsonen, S., and RosQvisT, T. (1991): 'Validated method for automatic detection of lung sound crackles', Med. Biol. Eng. Comput., 29, pp. 517-521

KOMPIS, M. (1995): 'Entwicklung und Evaluation eines elektronischen Lungengeräuschsimulators' (Doctoral thesis, School of Medicine, University of Zurich, Switzerland)

KraivaN, S. S. (1986): 'Effects of lung volume and aiflow on the frequency spectrum of vesicular lung sounds', Respir. Physiol., 66, pp. $1-9$

Kraman, S. S., Pasterkamp, H., Kompis, M., TaKase, M., and WoDICKA, G. R. (1996): 'Origin and significance of tracheal sound spectral analysis'. Proc. Int. Lung Sound Conf. Chester, UK, p. 2

MANGione, S., NiEMANN, L. Z., and Graceley, E. A. (1992): 'A comparison of computer-based leaming (CBL) and small group teacking of pulmonary auscultation to first year medical students'. Proc. Int. Lung Sound Conf., Helsinki, Finland, p. 39

Munakata, M., UKTTA, H., DoI, I., OHTSUKA, Y., MASAKI, Y., HOMMA, Y., and KAWAKAM, Y. (1991): 'Spectral and waveform characteristics of fine and coarse crackles', Thorax, 46, pp. 651657

NisSAN, M., and GaVRIELY, N. (1993): 'A microcomputer based lung sounds analysis', Comp. Methods Programs Biomed., 40, pp. 7-13

Pasterkamp, H., Carson, C., Daren, D., and $O H, Y$. (1989): 'Digital respirosonography-new images of lung sounds', Chest, 96, pp. $1405-1412$ 
PASTERKAMP, H. (1992): 'Nevie Entwicklungen und Erkenntnisse bei der Erforschung von Atemgeräuschen', Pneumologie, 46, pp. 602611

RUSso, A. G. (1991): 'RALE (respiration acoustics laboratory environment)', Focus Crit. Care, 18, pp. 527

SANKuR, B., KaYHA, Y. P., GULER, E. C., and ENGIN, T. (1994): 'Comparison of AR-based algorittm for respiratory sounds classification', Comput. Biol. Med, 24, pp. 67-76

SEstim, P., RENzoni, E., Rossi, M., Beltravi, V., and VagliasinDr, M. (1995): 'Multimedia presentation of lung sounds as a leaming aid for medical students', Eur. Respir. J., 8, pp. 783 788

TRAN, T., JONES, N. B., and FOTHERGiLl, J. C. (1995): 'Heart sound simulator", Med. Biol. Eng. Comput., 33, pp. 357-359

VANDERSChOOT, J., NiLS, G. J., VAN dE COPELLO, K., and SChrelR, H. J. W. (1992): 'AR model orders of lung sounds'. Proc. Annu. Int. Conf. IEEE. Eng. Med. Biol. Soc., 14, pp. 2531-2532
WIDROW, B.. and STEARtiS, S. D. (1985): 'Adaptive signal processing' (Prentice-Hall) pp. 452-467

\section{Author's biography}

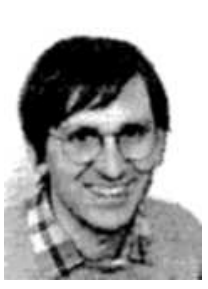

Martin Kompis received his diploma and doctoral degree in Electrical Engineering from the Swiss Federal Institute of Technology (ETH), Zurich, in 1989 and 1993, and his diploma in Medicine and MD-degree from the University of Zurich in 1994 and 1995, respectively. He is currently with the ENT department of the University Hospital of Berne, Switzerland. His research interests include biomedical acoustics and signal processing. 